\title{
A varying-gain ZNN model with fixed-time convergence and noise-tolerant performance for time-varying linear equation and inequality systems
}

This paper was downloaded from TechRxiv (https://www.techrxiv.org).

\section{LICENSE}

CC BY 4.0

SUBMISSION DATE / POSTED DATE

$11-11-2021 / 16-11-2021$

\section{CITATION}

Zhang, Miaomiao (2021): A varying-gain ZNN model with fixed-time convergence and noise-tolerant performance for time-varying linear equation and inequality systems. TechRxiv. Preprint. https://doi.org/10.36227/techrxiv.16988404.v1

$\mathrm{DOI}$ 


\title{
A varying-gain $\mathrm{ZNN}$ model with fixed-time convergence and noise-tolerant performance for time-varying linear equation and inequality systems
}

\author{
Miaomiao Zhang and Bing Zheng
}

\begin{abstract}
In this paper, a varying-gain zeroing (or Zhang) neural network (VG-ZNN) is proposed to obtain the online solution of the time-varying linear equation and inequality system. Distinguished from the fixed-value design parameter in the original zeroing (or Zhang) neural network ( $\mathrm{ZNN}$ ) models, the design parameter of the VG-ZNN model is a nonlinear function that changes with time. The VG-ZNN model composed of the new time-varying design parameter we proposed can achieve fixedtime convergence and tolerate time-varying bounded noise and time-varying derivable noise. The theoretical detailed analysis of the convergence and robustness of the VG-ZNN model are given. Numerical experiments are performed to confirm the theoretical results, including numerical experiments of proposed VG-ZNN model and numerical comparison with the original $\mathrm{ZNN}$ models and the known VG-ZNN models. Furthermore, the VG-ZNN model is also applied to the control of six-link robot manipulator, thus showing the applicability of the proposed VG-ZNN model.
\end{abstract}

Index Terms-varying-gain zeroing neural network, timevarying system, fixed-time convergence, noise-tolerant, mobile manipulator control.

\section{INTRODUCTION}

$\mathbf{S}$ OLVING the systems of linear equation and inequality arises in numerous fields of science, engineering, and business, such as image restoration, computer tomography, system identification, and control system synthesis, all of these require solving a large linear equation and inequality system within a reasonable time frame [1]-[5]. There are some methods for solving it. For example, Castillo et al. proposed a Gamma algorithm in [6], abstract algorithm proposed by Spedicato et al. [7] and its variants [8], [9], and some recurrent neural networks (RNNs) [10]-[12]. They all can solve the linear equation and inequality system effectively, but it should be pointed out that these methods are proposed for solving static linear equation and inequality system and in current practical applications, the problem is always related to time. Therefore, the methods of solving the time-varying linear equation and inequality system is most in need of discussion.

At present, one of the most effective methods for solving time-varying problems is zeroing/Zhang neural network (ZNN) proposed by Zhang et al. in [13], such as [14]-[16]. Just like its name, this model uses an appropriate activation function to make each element of the matrix/vector valued

This work is supported by the National Natural Science Foundation of China (Grant No.12071196). (Corresponding authors: Bing Zheng).

Miaomiao Zhang and Bing Zheng are with the School of Mathematics and Statistics, Lanzhou University, Lanzhou 730000, China (e-mail: mmzhang19@1zu.edu.cn and bzheng@1zu.edu.cn). error function converge to zero to achieve the purpose of solving the problem [17]. For example, Zhang et al. proposed the original ZNN model which can achieve exponential convergence to solve the time-varying matrix inversion in [18] and $\mathrm{Li}$ et al. proposed a finite-time ZNN model which activated by the sign-bi-power function and can achieve finitetime convergence to solve the time-varying Sylvester equation in [19], [20]. It is very necessary to point out that although the finite-time ZNN model improves the convergence time from infinite to finite, the upper bound of the convergence time is related to the initial value. That is to say, when the initial value is larger, the convergence time of the ZNN model may be longer [21]. Therefore, the main problem now is to eliminate the influence of the initial value on the upper bound of the convergence time. On the other hand, in the application of ZNN model, noise is inevitable, so the noisetolerant performance of $\mathrm{ZNN}$ models is another problem that needs to be solved. In fact, in order to solve the noise problem, Jin $e t$ al. once proposed a noise-tolerant ZNN model to solve the time-varying matrix inversion in [22], but the upper bound of the convergence time of this $\mathrm{ZNN}$ model is still related to the initial value. In fact, the above two problems were solved at the same time in [23], Xiao et al. proposed a novel ZNN model that can achieve fixed-time convergence and noise-tolerant performance to solve the time-varying Sylvester equation. In other words, the novel ZNN model has a clear upper bound on the convergence time which is only related to the parameters in the model and in [24], Zhang et al. generalized this ZNN model.

Although the proposed ZNN models in [23], [24] can achieve fixed-time convergence and noise-tolerant performance, their design parameter is still fixed. That is to say, in practical applications, the design parameter need to be set as large as possible, so that the convergence time will be as short as possible, but this is difficult to implement in actual systems. Besides, in practical applications, the convergence parameters are always related to the reciprocal of capacitive parameters and inductance parameters, i.e., the convergence parameters change with time in the hardware system [25]. To this end, Zhang et al. proposed a varying-gain ZNN (VGZNN) model with design parameter as power function in [26] and exponential function in [27]. However, although the above two VG-ZNN models used time-varying design parameter, they still did not solve the two problems we mentioned above. Therefore, Xiao et al. improved the VG-ZNN model whose design parameter is exponential function, and proved that 
this VG-ZNN model can achieve fixed-time convergence and noise-tolerant performance in [28].

In this paper, inspired by [26]-[28], we proposed a new time-varying design parameter and proved that the resulting VG-ZNN model can achieve fixed-time convergence and noise-tolerant performance. It can also be regarded as the promotion of the VG-ZNN model proposed by Xiao et al. in [28]. The numerical experiments are performed to confirm the superiority of our VG-ZNN model over the known VGZNN models and original ZNN model proposed in [29] when solving the time-varying linear equation and inequality system. To the beat of our knowledge, this is the first time a VGZNN model has been used to solve this problem. Moreover, we apply the proposed VG-ZNN model to the control of robot manipulator. Physical experiment performed on a sixlink planar robot manipulator is presented to demonstrate physical realizability and effectiveness of the proposed VGZNN model.

\section{ZNN MODELS}

In this section, we first present the original $\mathrm{ZNN}$ model for solving time-varying linear equations and inequality systems, and then propose a new time-varying design parameter to form a VG-ZNN model for solving the same system.

\section{A. Original ZNN model}

In this paper, we consider the following time-varying linear equation and inequality system:

$$
\left\{\begin{array}{l}
A(t) x(t)=b(t) \\
C(t) x(t) \leq d(t)
\end{array}\right.
$$

where $A(t) \in \mathbb{R}^{m \times n}$ (with $m<n$ ), $C(t) \in \mathbb{R}^{p \times n}$ (with $p<n), b(t) \in \mathbb{R}^{m}$, and $d(t) \in \mathbb{R}^{p}$ are known timevarying coefficient matrices and vectors, and $x(t) \in \mathbb{R}^{n}$ is the unknown vector to be obtained. In order to ensure the existence of the solution, we assume that $A(t)$ and $C(t)$ are row-full-rank matrices.

From [29], the equation (1) can be transformed into the following form by introducing a non-negative time-varying vector $y^{2}(t)=\left[y_{1}^{2}(t), \cdots, y_{p}^{2}(t)\right]^{T}=D(t) y(t)$ with $y(t)=$ $\left[y_{1}(t), y_{2}(t), \cdots, y_{p}(t)\right]^{T}$ and $D(t)=\operatorname{diag}\{y(t)\}$

$$
\left\{\begin{array}{c}
A(t) x(t)-b(t)=0 \\
C(t) x(t)-d(t)+y^{2}(t)=0 .
\end{array}\right.
$$

It can be sorted into the following equation

$$
M(t) u(t)-p(t)=0
$$

where the coefficient matrix $M(t) \in \mathbb{R}^{(m+p) \times(n+p)}$, vector $p(t) \in \mathbb{R}^{m+p}$, and the unknown vector $u(t)$ are defined as

$$
M(t)=\left(\begin{array}{cc}
A(t) & 0 \\
C(t) & D(t)
\end{array}\right), p(t)=\left(\begin{array}{c}
b(t) \\
d(t)
\end{array}\right)
$$

and $u(t)=[x(t) ; y(t)]$.

To make each element of the error function going to zero with time, we consider the following ZNN model formula with the error function $e(t)=M(t) u(t)-p(t)$

$$
\dot{e}(t)=-\beta \mathcal{F}(e(t))
$$

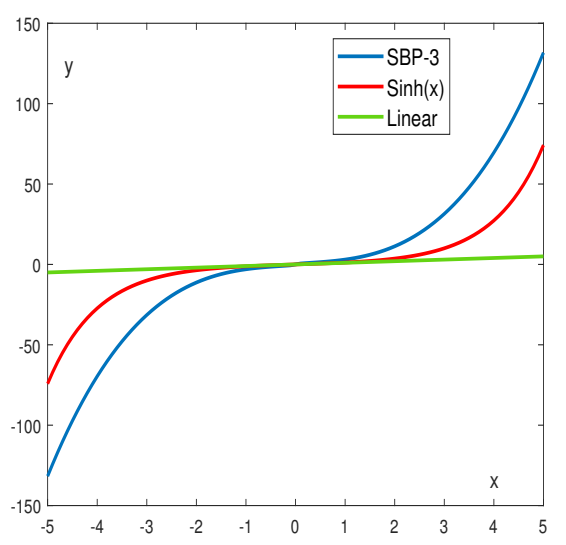

Fig. 1. Sign-bi-power function with $k_{1}=k_{2}=k_{3}=1, \eta=1 / 3$, and $\omega=3$ and hyperbolic-sine function with $\iota=1$.

where $\dot{e}(t)$ denotes the time derivative of $e(t), \beta>0$ is a fixed design parameter, and $\mathcal{F}(\cdot): \mathbb{R}^{m+p} \rightarrow \mathbb{R}^{m+p}$ denotes a vector-valued activation function array. Taking the derivative of the error function $e(t)$ and then substituting it into the design formula yields the following original $\mathrm{ZNN}$ model for the time-varying linear equation and inequality system:

$$
\dot{u}(t)=-W^{\dagger}(t)(Q(t) u(t)-\dot{p}(t)+\beta \mathcal{F}(M(t) u(t)-p(t)))
$$

with the coefficient matrices $W(t) \in \mathbb{R}^{(m+p) \times(n+p)}$ and $Q(t) \in \mathbb{R}^{(m+p) \times(n+p)}$ are defined as

$$
W(t)=\left(\begin{array}{cc}
A(t) & 0 \\
C(t) & 2 D(t)
\end{array}\right), Q(t)=\left(\begin{array}{cc}
\dot{A}(t) & 0 \\
\dot{C}(t) & 0
\end{array}\right),
$$

and $W^{\dagger}(t)$ is the pseudoinverse of $W(t)$.

For the original ZNN model (2), different kinds of activation function will lead to different performance, such as linear function, hyperbolic-sine function, and sign-bi-power function. The specific form of those three activation function is given below.

1) Linear function: $y=x$;

2) Hyperbolic-sine function: $y=\left(e^{\iota x}+e^{-\iota x}\right) / 2$, with $\iota>$ 0 ;

3) Sign-bi-power function: $y=\left(k_{1}|x|^{\eta}+k_{2}|x|^{\omega}\right) \operatorname{sign}(x)+$ $k_{3} x$, with $k_{1}, k_{2}$, and $k_{3}>0,0<\eta<1$, and $\omega>1$.

However, we still can not find the optimal activation function. The next lemma [27] will tell us how to choose the activation function that can make the $\mathrm{ZNN}$ model have a shorter convergence time.

Lemma 1 In order to compare the convergence time of two systems $M$ and $N$, taking Lyapunov functions $V_{M}(t) \geq 0$ and $V_{N}(t) \geq 0$, and the time derivatives $\dot{V}_{M}(t)<0$ and $\dot{V}_{N}(t)<0$ into consideration, if the derivative $\dot{V}_{M}(t)$ and $\dot{V}_{N}(t)$ satisfy $0>\dot{V}_{M}(t)>\dot{V}_{N}(t)$, then starting from the same initial value $E_{M}(0)=E_{N}(0)$ of the question, the convergence time of $V_{M}(t)$ is longer than that of $V_{N}(t)<0$.

From Lemma 1 and Fig. 1, it can seen that the sign-bi-power function $\left(k_{1}=k_{2}=k_{3}=1, \eta=1 / 3\right.$, and $\left.\omega=3\right)$ is better 
than the hyperbolic-sine function $(\iota=1)$ and the hyperbolicsine function $(\iota=1)$ is better than the linear function. Thus, the sign-bi-power function is used in this paper.

\section{B. Varying-gain ZNN Models}

In order to solve the problem that the design parameter of the original $\mathrm{ZNN}$ model is fixed, $\mathrm{ZNN}$ model with timevarying design parameter has begun to be studied. According to the varying-gain $\mathrm{ZNN}$ (VG-ZNN) model theory, the following design formula can be given:

$$
\dot{e}(t)=-\beta \nu(t) \mathcal{F}(e(t))
$$

where $\nu(t)$ is a time-varying smooth nonlinear function (linear function can be used as special cases) and $\nu(t)>0$ for $t \in$ $[0,+\infty)$. Hence, the VG-ZNN model for time-varying linear equation and inequality can be obtained:

$\dot{u}(t)=-W^{\dagger}(t)(Q(t) u(t)-\dot{p}(t)+\beta \nu(t) \mathcal{F}(M(t) u(t)-p(t)))$.

For the setting of the time-varying design parameter, Zhang et al. first proposed a power function design parameter

$$
\varphi_{1}(t)=t^{\alpha}+\alpha
$$

where $\alpha>0$ in [26] and then Xiao et al. proposed an exponential function design parameter

$$
\varphi_{2}(t)=e^{\lambda_{1} \operatorname{arccot}(t)+\lambda_{2} t}
$$

with $\lambda_{1}>0$, and $\lambda_{2}>0$ in [28].

However, according to the nature of the power function $f(t)=t^{\alpha}, \alpha>1$, the value of the function in $t \in[0,1]$ is much smaller than $t$. In other words, when $\alpha>1$, the time-varying design parameter $\varphi_{1}(t)$ does not perform well. On the other hand, from Lemma 1 , when $\nu(t)>1$, the VGZNN model (3) can converge faster than the original ZNN model (2). Thus, inspired by $\varphi_{1}(t)$ and $\varphi_{2}(t)$, the following new time-varying design parameter is given:

$$
\varphi(t)=\left\{\begin{array}{cr}
t^{r}+\frac{1}{r}, & 0<r \leq 1 \\
r^{\left(\lambda_{1} \operatorname{arccot}(t)+\lambda_{2} t\right)}, & r>1
\end{array}\right.
$$

where $\lambda_{1}, \lambda_{2}>0$.

\section{TheORETICAL ANALYSIS AND RESUlTS}

This section presents the theoretical analysis and results of the proposed VG-ZNN model (3) on solving time-varying linear equation and inequality system, including convergence time analysis and robustness analysis.

\section{A. Fixed-time convergence of the VG-ZNN model}

Theorem 1 Given the time-varying linear equation and inequality system (1), if the VG-ZNN model (3) activated by the sign-bi-power function is used, then the state matrix $x(t)$ starting from any initial state $x(0)$ always converges to a time-varying theoretical solution $x^{*}(t)$. In particular, when the design parameter is set to $\varphi(t)$, the VG-ZNN model can converge in the fixed time

$$
t_{c} \leq\left\{\begin{array}{cl}
{\left[\frac{1+r}{\beta k_{2}(\omega-1)}+\frac{1+r}{\beta k_{1}(1-\eta)}\right]^{\frac{1}{1+r}},} & \text { if } r \leq 1, \\
\frac{1}{\lambda_{2} \ln (r)} \ln \left(1+\frac{\lambda_{2} \ln (r)}{\beta k_{2}(\omega-1)}+\frac{\lambda_{2} \ln (r)}{\beta k_{1}(1-\eta)}\right), & \text { if } r>1
\end{array}\right.
$$

where the parameters $\beta, r, k_{1}, k_{2}, \eta, \omega$, and $\lambda_{2}$ are the same as before.

Proof Recall $\dot{e}(t)=-\beta \nu(t) \mathcal{F}(e(t))$. We define a Lyapunov function $v_{i}(t)=\left|e_{i}(t)\right| \geq 0$ for the $i$-th element of $e(t)$ and $i \in\{1,2, \cdots, m+p\}$. Its time derivative is

$$
\begin{aligned}
\dot{v}_{i}(t) & =\dot{e}_{i}(t) \operatorname{sign}\left(e_{i}(t)\right) \\
& =-\beta \nu(t)\left(k_{1}\left|e_{i}(t)\right|^{\eta}+k_{2}\left|e_{i}\right|^{\omega}+k_{3}\left|e_{i}(t)\right|\right) \\
& =-\beta \nu(t)\left(k_{1} v_{i}(t)^{\eta}+k_{2} v_{i}^{\omega}(t)+k_{3} v_{i}(t)\right) \\
& \leq 0 .
\end{aligned}
$$

Therefore, based on the Lyapunov stability theory [30], $e_{i}(t)$ globally converges to zero with time for each $i$. In other words, the error matrix $e(t)$ globally converges to zero with time.

Next, let us probe the convergence time of the VG-ZNN model composed of the time-varying design parameter $\varphi(t)$. We first prove the conclusion when $r \leq 1$.

From (4), the following equation can be got:

$$
\begin{aligned}
\dot{v}_{i}(t) & =-\beta\left(t^{r}+1 / r\right)\left(k_{1} v_{i}(t)^{\eta}+k_{2} v_{i}^{\omega}(t)+k_{3} v_{i}(t)\right) \\
& \leq-\beta t^{r}\left(k_{1} v_{i}(t)^{\eta}+k_{2} v_{i}^{\omega}(t)+k_{3} v_{i}(t)\right)
\end{aligned}
$$

In order to study the convergence time of the VG-ZNN model, proceed in the following two steps:

Step 1. When $\left|e_{i}(0)\right|>1 \rightarrow\left|e_{i}\left(t_{1}\right)\right|=1$. From the equation (5), it follows that

$$
\dot{v}_{i}(t) \leq-\beta t^{r} k_{2} v_{i}^{\omega}(t)
$$

i.e.,

$$
v_{i}^{-\omega}(t) d v_{i}(t) \leq-\beta k_{2} t^{r} d t
$$

Integrating both sides of the equation (6) from 0 to $t_{1}$, we get

$$
\begin{aligned}
\int_{v_{i}(0)}^{v_{i}\left(t_{1}\right)} v_{i}^{-\omega}(t) d v_{i}(t) & \leq \int_{0}^{t_{1}}-\beta k_{2} t^{r} d t \\
\left.\frac{1}{1-\omega} v_{i}^{1-\omega}(t)\right|_{v_{i}(0)} ^{1} & \leq-\left.\frac{\beta k_{2}}{1+r} t^{1+r}\right|_{0} ^{t_{1}} \\
\frac{1}{1-\omega}\left(1-v_{i}^{1-\omega}(0)\right) & \leq-\frac{\beta k_{2}}{1+r} t_{1}^{1+r} .
\end{aligned}
$$

It is easy to get time $t_{1}$ to satisfy:

$$
\begin{aligned}
t_{1} & \leq\left[\frac{(1+r)\left(1-v_{i}^{1-\omega}(0)\right)}{\beta k_{2}(\omega-1)}\right]^{\frac{1}{1+r}} \\
& \leq\left[\frac{1+r}{\beta k_{2}(\omega-1)}\right]^{\frac{1}{1+r}} .
\end{aligned}
$$

Step 2. When $\left|e_{i}\left(t_{1}\right)\right|=1 \rightarrow\left|e_{i}\left(t_{1}+t_{2}\right)\right|=0$, the inequality (5) is equal to

$$
\dot{v}_{i}(t) \leq-\beta t^{r} k_{1} v_{i}^{\eta}(t)
$$

i.e.,

$$
v_{i}^{-\eta}(t) d v_{i}(t) \leq-\beta k_{1} t^{r} d t .
$$


Integrating both sides of the equation (7) from $t_{1}$ to $t_{1}+t_{2}$, we get

$$
\begin{aligned}
& \int_{v_{i}\left(t_{1}\right)}^{v_{i}\left(t_{1}+t_{2}\right)} v_{i}^{-\eta}(t) d v_{i}(t) \leq \int_{t_{1}}^{t_{1}+t_{2}}-\beta k_{1} t^{r} d t \\
& \left.\frac{1}{1-\eta} v_{i}^{1-\eta}(t)\right|_{v_{i}\left(t_{1}\right)} ^{v_{i}\left(t_{1}+t_{2}\right)} \leq-\left.\frac{\beta k_{1}}{1+r} t^{1+r}\right|_{t_{1}} ^{t_{1}+t_{2}} \\
& \quad-\frac{1}{1-\eta} \leq-\frac{\beta k_{1}}{1+r}\left(\left(t_{1}+t_{2}\right)^{1+r}-t_{1}^{1+r}\right) \\
& \left(t_{1}+t_{2}\right)^{1+r} \leq t_{1}^{1+r}+\frac{1+r}{\beta k_{1}(1-\eta)} .
\end{aligned}
$$

Then, the time $t_{c}$ is easily obtained:

$$
\begin{aligned}
t_{c} & =t_{1}+t_{2} \\
& \leq\left[\frac{(1+r)\left(1-v_{i}^{1-\omega}(0)\right)}{\beta k_{2}(\omega-1)}+\frac{1+r}{\beta k_{1}(1-\eta)}\right]^{\frac{1}{1+r}} \\
& \leq\left[\frac{1+r}{\beta k_{2}(\omega-1)}+\frac{1+r}{\beta k_{1}(1-\eta)}\right]^{\frac{1}{1+r}},
\end{aligned}
$$

i.e., the error function $e(t)$ globally converges to zero in the fixed-time $t_{c}$ when $r \leq 1$.

The proof of the conclusion when $r>1$ is similar to when $r \leq 1$. But for the completeness, here we also give its proof. From (4), we have

$$
\begin{aligned}
\dot{v}_{i}(t) & =-\beta r^{\left(\lambda_{1} \operatorname{arccot}(t)+\lambda_{2} t\right)}\left(k_{1} v_{i}(t)^{\eta}+k_{2} v_{i}^{\omega}(t)+k_{3} v_{i}(t)\right) \\
& \leq-\beta r^{\lambda_{2} t}\left(k_{1} v_{i}(t)^{\eta}+k_{2} v_{i}^{\omega}(t)+k_{3} v_{i}(t)\right)
\end{aligned}
$$

Next studying the convergence time of the VG-ZNN model in two steps:

Step 1. When $\left|e_{i}(0)\right|>1 \rightarrow\left|e_{i}\left(t_{1}\right)\right|=1$, the inequality (8) is equal to

$$
\dot{v}_{i}(t) \leq-\beta k_{2} r^{\left(\lambda_{2} t\right)} v_{i}^{\omega}(t)
$$

i.e.,

$$
v_{i}^{-\omega(t)} d v_{i}(t) \leq-\beta k_{2} r^{\left(\lambda_{2} t\right)} d t .
$$

Integrating both sides of the equation from 0 to $t_{1}$, the time $t_{1}$ is easily obtained:

$$
\begin{aligned}
t_{1} & \leq \frac{1}{\lambda_{2} \ln (r)} \ln \left(1+\frac{\lambda_{2} \ln (r)\left(1-v_{i}^{1-\omega(0)}\right)}{\beta k_{2}(\omega-1)}\right) \\
& \leq \frac{1}{\lambda_{2} \ln (r)} \ln \left(1+\frac{\lambda_{2} \ln (r)}{\beta k_{2}(\omega-1)}\right) .
\end{aligned}
$$

Step 2. When $\left|e_{i}\left(t_{1}\right)\right|<1 \rightarrow\left|e_{i}(t 2)\right|=0$, inequality (8) is equal to

$$
\dot{v}_{i}(t) \leq-\beta k_{1} r^{\left(\lambda_{2} t\right)} v_{i}^{\eta}(t)
$$

i.e.,

$$
v_{i}^{-\omega}(t) d v_{i}(t) \leq-\beta k_{1} r^{\left(\lambda_{2} t\right)} d t .
$$

Integrating both sides of the equation from 0 to $t_{1}$, the time $t_{c}$ is easily obtained:

$$
\begin{aligned}
t_{c} & =t_{1}+t_{2} \\
& \leq \frac{1}{\lambda_{2} \ln (r)} \ln \left(1+\frac{\lambda_{2} \ln (r)\left(1-v_{i}^{1-\omega(0)}\right)}{\beta k_{2}(\omega-1)}+\frac{\lambda_{2} \ln (r)}{\beta k_{1}(1-\eta)}\right) \\
& \leq \frac{1}{\lambda_{2} \ln (r)} \ln \left(1+\frac{\lambda_{2} \ln (r)}{\beta k_{2}(\omega-1)}+\frac{\lambda_{2} \ln (r)}{\beta k_{1}(1-\eta)}\right),
\end{aligned}
$$

i.e., the error function $e(t)$ globally converges to zero in the fixed-time $t_{c}$ when $r>1$. The proof is thus completed.

It should be noted that, according to the nature of the exponential function $f(x)=r^{x}, r>1$, as $r$ increases, the faster the growth rate of the function will be. Combined with the lemma 1, the convergence time of the constructed VG$\mathrm{ZNN}$ model will be shorter. We will verify this conclusion in the section (IV).

Remark 1 Using the proof method of theorem 1, it is easy to get that the original ZNN model (2) activated by the sign-bipower function also can achieve fixed-time convergence and the fixed convergence time is

$$
\frac{1}{\beta k_{2}(\omega-1)}+\frac{1}{\beta k_{1}(1-\eta)} .
$$

This is consistent with the conclusion in [23], [24].

\section{B. Noise-tolerant performance of the VG-ZNN model}

In this subsection, we are going to prove that the VGZNN model can tolerate two types of noise, the time-varying bounded noise and the time-varying derivable noise. Supposing $\Delta n(t)$ is a time-varying noise with vector value, we further investigate the following VG-ZNN model

$$
\begin{aligned}
\dot{u}(t)=- & W^{\dagger}(t)(Q(t) u(t)-\dot{p}(t)+\beta \nu(t) \\
& \mathcal{F}(M(t) u(t)-p(t)-\Delta n(t)) .
\end{aligned}
$$

Theorem 2 If $\Delta n(t)$ is a time-varying noise, then we have the following conclusions:

1) If the noise $\Delta n(t)$ is a time-varying bounded noise and the time-varying design parameter $\nu(t)$ satisfies $\lim _{t \rightarrow+\infty} \nu(t)=+\infty$, then the error of the VG-ZNN model composed of $\nu(t)$ can always converge to zero when time tends to infinity.

2) If the noise $\Delta n(t)$ is a time-varying noise with $\kappa$-order derivability and its derivative satisfies that $\lim _{t \rightarrow+\infty} \Delta n_{i}^{(j)}(t)=\infty, j \in\{0,1,2, \cdots, \kappa-1\}$ and $\left|\Delta n_{i}^{(\kappa)}(t)\right| \in(0, \zeta] \quad(\kappa$ is a positive integer and $\zeta$ is a nonzero constant), then the error of the $V G$ ZNN model whoes time-varying design parameter $\nu(t)$ is $\gamma$-order derivable $(\gamma>\kappa)$ and $\lim _{t \rightarrow+\infty} \nu^{(j)}(t)=$ $\infty, j \in\{0,1,2, \cdots, \kappa\}$ can always converge to zero when time tends to infinity. Moreover, if the timevarying noise $\Delta n(t)$ satisfies $\lim _{t \rightarrow+\infty} \Delta n_{i}(t)=\infty$ and $\lim _{t \rightarrow+\infty} \Delta n_{i}^{(j)}(t)=0, j \in\{1,2, \cdots, \kappa\}$, then this $V G$ ZNN model also can converge.

Proof Through the theory of the ZNN model, the equation (9) is equal to

$$
\dot{e}(t)=-\beta \nu(t) \mathcal{F}(e(t))+\Delta n(t) .
$$

In order to study the convergence of $e(t)$, we can study the ith (with $i=1,2, \cdots, m+p$ ) differential equation separately:

$$
\dot{e}_{i}(t)=-\beta \nu(t) \mathcal{F}\left(e_{i}(t)\right)+\Delta n_{i}(t)
$$


Then, we define a new Lyapunov function $l_{i}(t)=\frac{1}{2}\left|e_{i}(t)\right|^{2} \geq 0$ and its derivative is

$$
\begin{aligned}
\dot{l}_{i}(t) & =e_{i}(t) \dot{e}_{i}(t) \\
& =e_{i}(t)\left(-\beta \nu(t) \mathcal{F}\left(e_{i}(t)\right)+\Delta n_{i}(t)\right) .
\end{aligned}
$$

There are two cases to discuss the size of $i_{i}(t)$ :

(i) If $i_{i}(t) \leq 0$, then $\left|e_{i}(t)\right|$ will gradually decrease to zero, i.e. the VG-ZNN model can tolerant the noise $\Delta n(t)$.

(ii) If $i_{i}(t)>0,\left|e_{i}(t)\right|$ is monotonically increasing, that is, $\left|e_{i}(t)\right|$ will gradually increase as $t$ increases and $e_{i}(t) \Delta n_{i}(t)>0$.

So we only need to discuss the case (ii), that is

$$
e_{i}(t)\left(-\beta \nu(t) \mathcal{F}\left(e_{i}(t)\right)+\Delta n_{i}(t)\right)>0
$$

On this basis, we can know

$$
\left|\Delta n_{i}(t)\right|>\left|\beta \nu(t) \mathcal{F}\left(e_{i}(t)\right)\right| \text {. }
$$

It is easy to know that $\left|-\beta \nu(t) \mathcal{F}\left(e_{i}(t)\right)+\Delta n_{i}(t)\right|$ will decrease as $\left|e_{i}(t)\right|$ increase, and $\left|e_{i}(t)\right|$ will stop increasing until $-\beta \nu(t) \mathcal{F}\left(e_{i}(t)\right)+\Delta n_{i}(t)=0$. So, when $t \rightarrow \infty,\left|e_{i}(t)\right|$ is bounded by

$$
0 \leq\left|e_{i}(t)\right| \leq\left|\mathcal{F}^{-1}\left(\frac{\Delta n_{i}(t)}{\beta \nu(t)}\right)\right|,
$$

where $\mathcal{F}^{-1}(\cdot)$ is the inverse function of $\mathcal{F}(\cdot)$. Due to in this paper, the $\mathcal{F}(\cdot)$ is the sign-bi-power function, i.e., $|\mathcal{F}(x)| \geq$ $\left|k_{3} x\right|$, so $\left|\mathcal{F}^{-1}(x)\right| \leq\left|x / k_{3}\right|$.

Thus, the bound of $\left|e_{i}(t)\right|$ can be expressed as follows:

$$
0 \leq\left|e_{i}(t)\right| \leq\left|\frac{\Delta n_{i}(t)}{\beta k_{3} \nu(t)}\right|
$$

Next, we will discuss two kinds of noise separately:

For the time-varying bounded noise, there is a constant $\epsilon$ such that $\Delta n_{i}(t) \leq \epsilon$. In this case, inequality (10) can be indicated as

$$
0 \leq\left|e_{i}(t)\right| \leq\left|\frac{\epsilon}{\beta k_{3} \nu(t)}\right|
$$

When time $t$ tends to infinity, since $\lim _{t \rightarrow+\infty} \nu(t)=\infty$, there is

$$
\lim _{t \rightarrow+\infty}\left|e_{i}(t)\right| \leq \lim _{t \rightarrow+\infty}\left|\frac{\epsilon}{\beta k_{3} \nu(t)}\right|=0
$$

Hence,

$$
\lim _{t \rightarrow+\infty}\|e(t)\|_{2}=\sqrt{\sum_{i=1}^{m+p} \lim _{t \rightarrow+\infty}\left|e_{i}(t)\right|^{2}}=0
$$

i.e., the VG-ZNN model (9) can tolerate the time-varying bound noise $\Delta n(t)$.

For the time-varying noise $\Delta n(t)$ with $\kappa$-order derivability and $\kappa$-order derivative $\lim _{t \rightarrow+\infty} \Delta n_{i}^{(j)}(t)=\infty, j \in$ $\{0,1,2, \cdots, \kappa-1\}$ and $\Delta n_{i}^{(\kappa)}(t) \in(0, \zeta]$ ( $\kappa$ ia s positive integer and $\zeta$ is a positive constant), if the time-varying design parameter $\nu(t)$ is $\gamma$-order derivable $(\gamma>\kappa)$ and $\lim _{t \rightarrow \infty} \nu^{(j)}(t)=\infty, j \in\{0,1, \cdots, \kappa\}$ then

$$
\begin{aligned}
\lim _{t \rightarrow+\infty}\left|e_{i}(t)\right| & \leq \lim _{t \rightarrow+\infty}\left|\frac{\Delta n_{i}(t)}{\beta k_{3} \nu(t)}\right| \\
& =\lim _{t \rightarrow+\infty}\left|\frac{\Delta n_{i}^{(\kappa)}(t)}{\beta k_{3} \varphi^{(\kappa)}(t)}\right| \\
& \leq \lim _{t \rightarrow+\infty}\left|\frac{\zeta}{\beta k_{3} \nu^{(\kappa)}(t)}\right| \\
& =0 .
\end{aligned}
$$

From formula (11), it can be eaisly obtained that if noise $\Delta n_{i}(t)$ satisfies $\lim _{t \rightarrow+\infty} \Delta n^{(i)}(t)=0, i \in\{1,2, \cdots, \kappa\}$, then inequalities still holds. Therefore,

$$
\lim _{t \rightarrow+\infty}\|e(t)\|_{2}=0
$$

i.e., the VG-ZNN model (9) can tolerate the time-varying derivale noise $\Delta n(t)$. The proof is thus completed.

From the theorem 2, it can be obtained that the VGZNN model composed of the time-varying design parameter $\varphi_{1}(t), \varphi_{2}(t)$, and $\varphi(t)$ all can tolerate the time-varying bound noise and the VG-ZNN model composed of the time-varying design parameter $\varphi_{2}(t)$ and $\varphi(t), r>1$ both can tolerate the time-varying derivable noise.

\section{Simulation VERIFICATION}

In this section, for illustrating the above conclusions, numerical simulations are compared to substantiate the superior performance of VG-ZNN model composed of the time-varying design parameter $\varphi(t)$ for time-varying linear equation and inequality system.

In particular, the time-varying design parameter of the VGZNN models are set to: if $r \leq 1, \varphi_{1}(t)=t^{1 / 4}+1 / 4 ; \varphi(t)=$ $t^{1 / 4}+4$; if $r>1, \varphi_{1}(t)=t^{2}+2 ; \varphi_{2}(t)=e^{\operatorname{arccot}(t)+t} ; \varphi(t)=$ $2^{\operatorname{arccot}(t)+t}$ and $\varphi(t)=4^{\operatorname{arccot}(t)+t}$. From the theorem 1, the fixed convergence time of the VG-ZNN model under the above time-varying design parameter $\varphi(t)$ is 2.0815s $(r=$ $1 / 4), 1.2548 \mathrm{~s}(r=2), 1.0986 \mathrm{~s}(r=e)$, and $0.9578 \mathrm{~s}(r=$ 4).

Considering the time-varying linear equation and inequality system with the following time-varying matrices:

$$
\begin{gathered}
A(t)=\left(\begin{array}{c}
3+\sin (3 t) \\
5+\cos (3 t) \\
7-\sin (3 t) \\
9+\sin (t)-\cos (3 t)
\end{array}\right)^{T}, b(t)=2+\sin (4 t)-\cos (4 t) \\
C(t)=\left(\begin{array}{cc}
\sin (2 t) & -\cos (2 t) \\
\cos (2 t) & \sin (2 t) \\
-\sin (2 t) & \cos (2 t) \\
-\cos (2 t) & -\sin (2 t)
\end{array}\right)^{T}, d(t)=\left(\begin{array}{c}
2 \sin (2 t) \\
3 \cos (2 t)
\end{array}\right) .
\end{gathered}
$$

The corresponding simulation results are shown in Fig. 2 Fig. 5. As illustrated in Fig. 2(a), when $r \leq 1$, starting from five different initial states randomly selected in $[-2,2]^{6 \times 1}$, the neural states $x(t)$ of the VG-ZNN model always change with time. In Fig. 2(b), the residual error $e(t)=\|M(t) u(t)-p(t)\|_{2}$ 


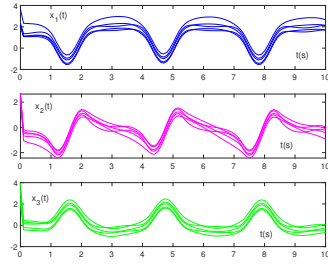

(a) State solution $x(t)$;

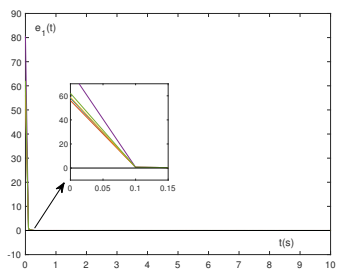

(c) $e_{1}(t)=A(t) x(t)-b(t)$;

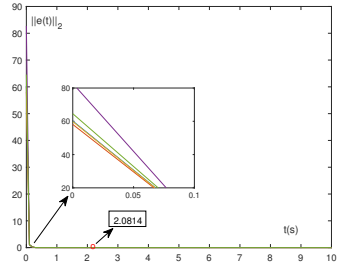

(b) $\|e(t)=W(t) u(t)-q(t)\|_{2}$;

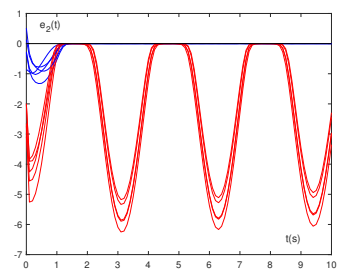

(d) $e_{2}(t)=C(t) x(t)-b(t)$

Fig. 2. Dynamic trajectories of the VG-ZNN model for solving the timevarying linear and inequality system.

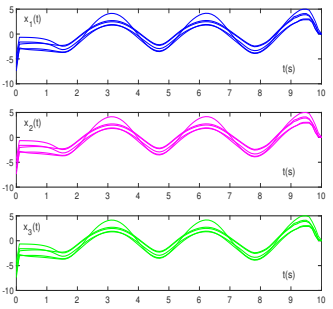

(a) State solution $x(t)$;

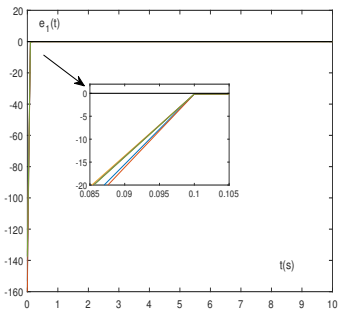

(c) $e_{1}(t)=A(t) x(t)-b(t)$;

Fig. 3. Dynamic trajectories of the VG-ZNN model for solving the timevarying linear and inequality system.

all can diminish to zero within the fixed convergence time which is shown by a red circle in the figure. In order to verify the accuracy of the results, we also give out two error figures. In Fig. 2(c), the error of linear equation $e_{1}(t)=A(t) x(t)-b(t)$ can always diminish zero, and in Fig. 2(d), each element of the error of inequality $e_{2}(t)=C(t) x(t)-d(t)$ is always less than or equal to 0 . Similarly, when $r>1$, the results are shown in Fig. 3 and due to the limited space, only the simulation result of $r=4$ is given.

Fig. 4 described the convergence behaviors of the residual error $\|e(t)\|_{2}$ of all VG-ZNN models composed of different time-varying design parameter $\nu(t)$ in the ideal environment, i.e., $\Delta n_{i}(t)=0$ and in the presence of some time-varying derivable noise. From Fig. 4(a), the error $\|e(t)\|_{2}$ generated

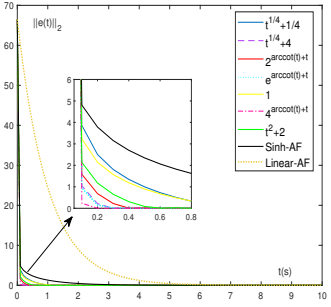

(a) $\Delta n_{i}(t)=0$;

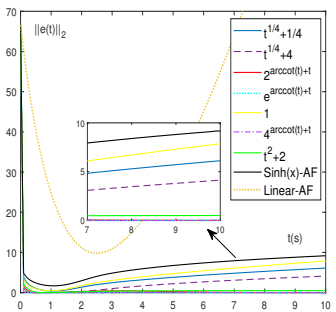

(c) $\Delta n_{i}(t)=t^{2}$;

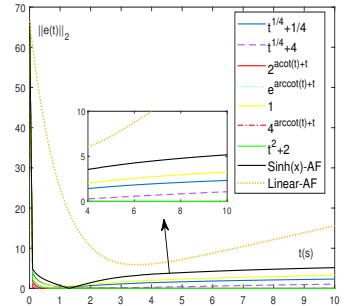

(b) $\Delta n_{i}(t)=t$;

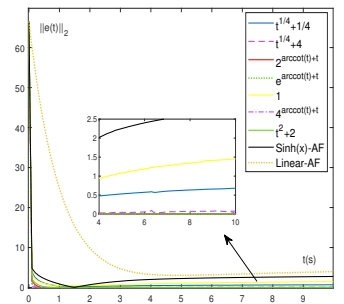

(d) $\Delta n_{i}(t)=\ln (1+t)$;
Fig. 4. Dynamic trajectories of error norms $\|e(t)\|_{2}$ by the VG-ZNN models and the original $\mathrm{ZNN}$ models for solving the time-varying linear and inequality system under various noises.

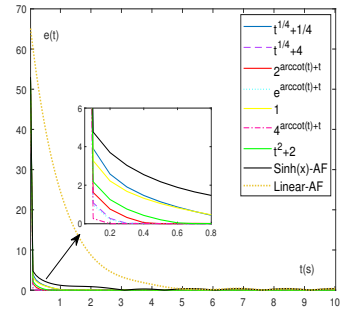

(a) $\Delta n_{i}(t)=0.5 \cos (2 t)$;

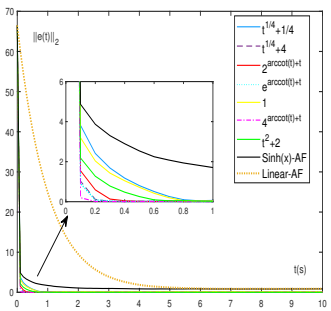

(c) $\Delta n_{i}(t) \in(0,1)$;

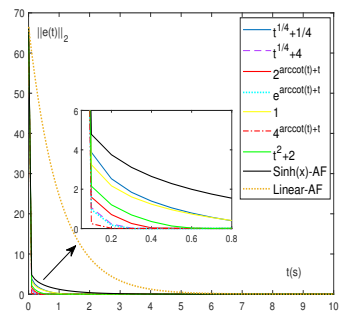

(b) $\Delta n_{i}(t)=0.2 e^{-t}$;

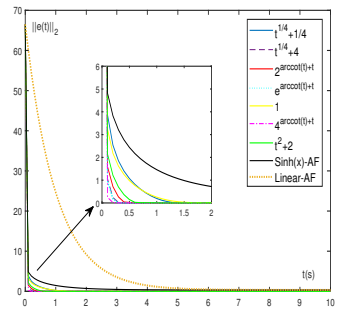

(d) $\Delta n_{i}(t)=0.5$;
Fig. 5. Dynamic trajectories of error norms $\|e(t)\|_{2}$ by the VG-ZNN models and the original ZNN models for solving the time-varying linear and inequality system under various noises.

by ZNN models all can diminish to zero, but the VG-ZNN models we proposed have a shorter convergence time. In addition, it can be seen from Fig. 4(b) - Fig. 4(d) that the error generated by the VG-ZNN models composed of $\varphi(t)$ with $r>1$ all can diminish to zero under the influence of time-varying derivable noise. Although the VG-ZNN model composed of $\varphi(t)=t^{1 / 4}+4$ can not tolerate the time-varying derivable noise, the error of the model is smaller than the VG-ZNN model composed of $\varphi_{1}(t)=t^{1 / 4}+1 / 4$. This shows that VG-ZNN model composed of $\varphi(t)$ has more advantages 


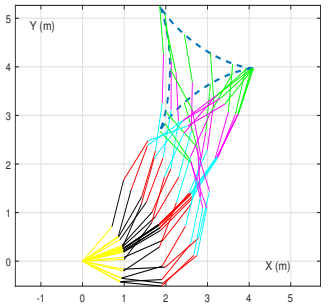

(a) Simulated motion trajectories;

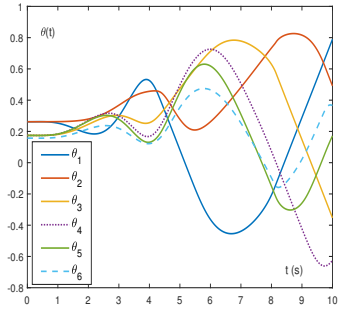

(c) Joint angle $\theta(t)$;

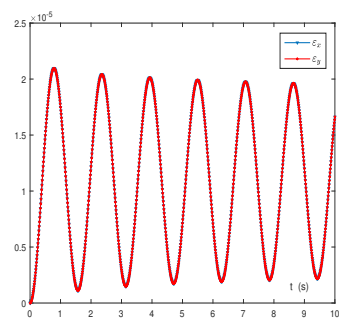

(b) End-effector tracking errors;

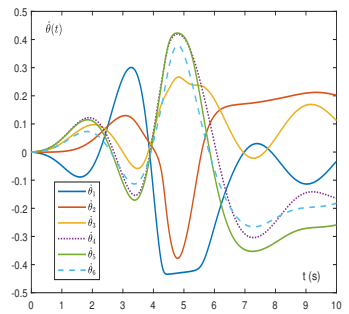

(d) Joint velocity $\dot{\theta}(t)$;
Fig. 6. Simulation results using the VG-ZNN model for the end-effector of six-link robot manipulator tracking a tricuspid path under the noise $\Delta n(t)=$ $0.2 \sin (4 t)$ when $r \leq 1$.

in the presence of noise. Moreover, it is worth noting that the error generated by the VG-ZNN model composed of $\varphi_{1}(t)=t^{2}+2$ also can diminish to zero under the influence of noise $\Delta n_{i}(t)=t$. This is because $\varphi_{1}(t)=t^{2}+2$ is 2 -order derivable and $\lim _{t \rightarrow+\infty} 2 t=+\infty$. It is also for this reason that in the presence of noise $\Delta n_{i}(t)=t^{2}$, this VG-ZNN model can not converge to zero. For the noise $\Delta n_{i}(t)=\ln (1+t)$, since $\lim _{t \rightarrow+\infty} \Delta n_{i}^{(j)}(t)=0, j \in\{1,2, \cdots,+\infty\}$, the error of the VG-ZNN models composed of $\varphi(t), r>1$ also can diminish to zero. Fig. 5 show that when the noise $\Delta n(t)$ is time-varying bounded, the VG-ZNN composed of $\varphi_{1}(t), \varphi_{2}(t)$, and $\varphi(t)$ all can diminish to zero.

From Fig. 4 and Fig. 5, it can be seen that the convergence time of VG-ZNN models composed of $\varphi(t), r>1$ becomes shorter as $r$ increases which verifies the conclusion we made earlier. Besides, regardless of the presence or absence of noise, the convergence performance of the original ZNN model activated by the sign-bi-power function is better than the linear or hyperbolic sine function, and the VG-ZNN model is better than the original $\mathrm{ZNN}$ model.

\section{Robot APPLICATION}

In this section, the proposed VG-ZNN model is employed to a six-link robot manipulator by solving the time-varying linear equation and inequality system.

The purpose of robot manipulator control can be achieved by solving the following time-varying linear equation with boundary constraints [29], [31]-[34]:

$$
\left\{\begin{aligned}
J(\theta(t)) \dot{\theta}(t) & =\dot{r}_{d}(t) \\
\dot{\theta}^{-} \leq \dot{\theta}(t) & \leq \dot{\theta}^{+} \\
\theta^{-} \leq \theta(t) & \leq \theta^{+}
\end{aligned}\right.
$$

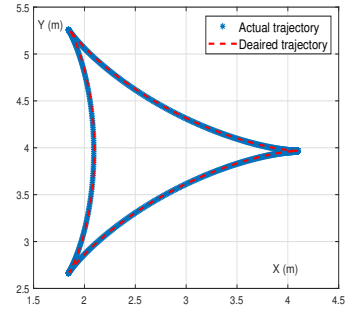

(a) Comparison of tracking trajectories;

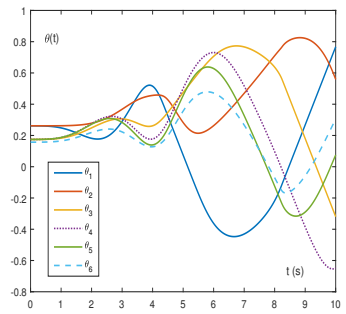

(c) Joint angle $\theta(t)$;

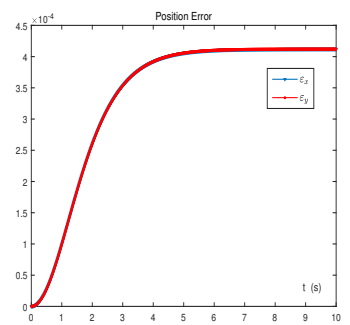

(b) End-effector tracking errors;

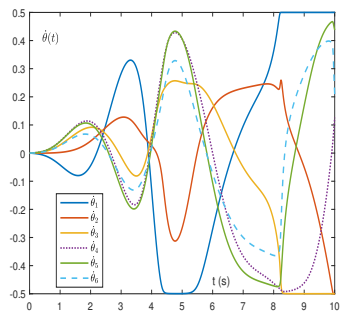

(d) Joint velocity $\dot{\theta}(t)$
Fig. 7. Simulation results using the VG-ZNN model for the end-effector of six-link robot manipulator tracking a tricuspid path under the noise $\Delta n(t)=$ $0.2 t$ when $r>1$.

where $\theta(t) \in \mathbb{R}^{n}$ and $\dot{\theta}(t) \in \mathbb{R}^{n}$ are joint-angle and jointvelocity vectors, respectively. $J(\theta(t)) \in \mathbb{R}^{m \times n}$ is the Jacobian matrix, $r_{d}(t) \in \mathbb{R}^{m}$ is the desired path with $\dot{r}_{d}(t)$ as its time derivative, and $\dot{\theta}^{ \pm}$and $\theta^{ \pm}$correspond to the limits of $\dot{\theta}(t)$ and $\theta(t)$.

In fact, it only need to make

$$
\begin{gathered}
A(t)=J(\theta(t)), b(t)=\dot{r}_{d}(t), \\
C(t)=\left(\begin{array}{c}
-I \\
I
\end{array}\right), d(t)=\left(\begin{array}{c}
-\max \left\{\dot{\theta}^{-}, \mu\left(\theta^{-}-\theta(t)\right)\right\} \\
\min \left\{\dot{\theta}^{+}, \mu\left(\theta^{+}-\theta(t)\right)\right\}
\end{array}\right),
\end{gathered}
$$

with $\mu>0 \in \mathbb{R}$, the equation (12) can be easily transformed into a time-varying linear equation and inequality system from [29], [34].

The proposed VG-ZNN model is simulated for the six-link robot manipulator with its end-effector tracking the tricuspid path in a 2-D plane and with the following physical limits:

$$
\begin{gathered}
\theta^{-}=-\theta^{+}=-[4 \pi / 3 ; 2 \pi / 3 ; 2 \pi / 3 ; 2 \pi / 3 ; \pi / 4 ; \pi / 4 ;]^{T} \mathrm{rad} \\
\dot{\theta}^{-}=-\dot{\theta}^{+}=-[0.5 ; 0.5 ; 0.5 ; 0.5 ; 0.5 ; 0.5]^{T} \mathrm{rad} / \mathrm{s} .
\end{gathered}
$$

The simulation results are shown in Fig. 6 and Fig. 7. Fig. 6 shows the whole tracking process of the mobile manipulator controlled by VG-ZNN model composed of the time-varying design parameter $\varphi(t)=t^{1 / 4}+4$ under the noise $\Delta n_{i}(t)=$ $0.2 \sin (4 t)$. Thereinto, Fig. 6(a) shows the entire space trajectory of the VG-ZNN model and the effective application of the VG-ZNN model on the six-link robot manipulator, that is, the end effector of the robot can complete the tracking task well when the error is not greater than $2.5 \times 10^{-5}$ from Fig. 6(b). Furthermore, Fig. 6(c) and Fig. 6(d) show that all joint variables $(\theta$ and $\dot{\theta})$ do not exceed their physical limits. Similarly, when the time-varying design parameter is set to $\varphi(t)=4^{\operatorname{arccot}(t)+t}$, the simulation results in Fig. 7. They also 
show that the VG-ZNN model also can track the trajectory well under the noise $\Delta n_{i}(t)=0.2 t$ with the tracking error of not more than $4.5 \times 10^{-4}$. These results verify the effective application of the proposed VG-ZNN model to six-link robot manipulator.

\section{CONCLUSION}

In this paper, a varying-gain $\mathrm{ZNN}$ model with fixed-time convergence and noise-tolerant performance is proposed to solve the liner equation and inequality system. Its fixed-time convergence and noise-tolerant properties were theoretically analyzed. Some numerical comparisons with the known VG$\mathrm{ZNN}$ models and the original ZNN models are also conducted. Moreover, we successfully apply the varying-gain ZNN model to robot manipulator control, which indicates the applicability of our new model in practice.

\section{REFERENCES}

[1] S. Agmon, "The relaxation method for linear inequalities," Canadian J. Math., vol. 6, pp. 382-392, 1954.

[2] C. Yair and E. Tommy, "New methods for linear inequalities," Linear Algebra and its Applications, vol. 42, pp. 199-211, 1982.

[3] R. Bramley and B. Winnicka, "Solving linear inequalities in a least squares sense," SIAM Journal on Scientific Computing, vol. 17, no. 1, p. 275-286, 1996.

[4] H. Li, J. Luo, and Q. Wang, "Solvability and feasibility of interval linear equations and inequalities," Linear Algebra and its Applications, vol. 463, pp. 78-94, 2014

[5] A. I. Golikov and Y. G. Evtushenko, "Regularization and normal solutions of systems of linear equations and inequalities," Proceedings of the Steklov Institute of Mathematics, vol. 289, no. 1, pp. 102-110, 2015.

[6] E. Castillo, F. Jubete, R. Pruneda, and C. Solares, "Obtaining simultaneous solutions of linear subsystems of inequalities and duals," Linear Algebra and its Applications, vol. 346, no. 1-3, pp. 131-154, 2002.

[7] E. Spedicato, Z. Xia, and L. W. Zhang, "ABS algorithms for linear equations and applications to optimization," journal of computational and applied mathematics, vol. 124, pp. 155-170, 2000.

[8] H. Esmaeili, N. Mahdavi-Amiri, and E. Spedicato, "Explicit ABS solution of a class of linear inequality systems and LP problems," Bull.iranian Math.soc, vol. 30, no. 2, pp. 21-38, 2004.

[9] E. Spedicato, E. Bodon, Z. Xia, and N. Mahdavi-Amiri, "ABS methods for continuous and integer linear equations and optimization," Central European Journal of Operations Research, vol. 18, pp. 73-95, 2010.

[10] A. Cichocki, J. Ramirez-Angulo, and R. Unbehauen, "Architectures for analog VLSI implementation of neural networks for solving linear equations with inequality constraints," in Proceedings. 1992 IEEE International Symposium on Circuits and Systems, vol. 3, 1992, pp. 15291532.

[11] Y. Xia, J. Wang, and D. L. Hung, "Recurrent neural networks for solving linear inequalities and equations," IEEE Transactions on Circuits and Systems I: Fundamental Theory and Applications, vol. 46, no. 4, pp. $452-462,1999$.

[12] X.-B. Liang and S. K. Tso, "Improved upper bound on step-size parameters of discrete-time recurrent neural networks for linear inequality and equation system," IEEE Transactions on Circuits and Systems I: Fundamental Theory and Applications, vol. 49, no. 5, pp. 695-698, 2002.

[13] Y. Zhang, D. Jiang, and J. Wang, "A recurrent neural network for solving Sylvester equation with time-varying coefficients," IEEE Transactions on Neural Networks, vol. 13, no. 5, pp. 1053-1063, 2002.

[14] W. Li, L. Xiao, and B. Liao, "A finite-time convergent and noiserejection recurrent neural network and its discretization for dynamic nonlinear equations solving," IEEE Transactions on Cybernetics, vol. 50, no. 7, pp. 3195-3207, 2020.

[15] Z. Zhang, Z. Li, and S. Yang, "A barrier varying-parameter dynamic learning network for solving time-varying quadratic programming problems with multiple constraints," IEEE Transactions on Cybernetics, pp 1-12, 2021, published online, doi=10.1109/TCYB.2021.3051261.
[16] M. Yang, Y. Zhang, N. Tan, M. Mao, and H. Hu, "7-instant discrete-time synthesis model solving future different-level linear matrix system via equivalency of zeroing neural network," IEEE Transactions on Cybernetics, pp. 1-10, 2021, published online, doi=10.1109/TCYB.2021.3051035

[17] Y. Zhang, B. Mu, and H. Zheng, "Link between and comparison and combination of Zhang neural network and quasi-newton BFGS method for time-varying quadratic minimization," IEEE Transactions on Cybernetics, vol. 43, no. 2, pp. 490-503, 2013.

[18] Y. Zhang and S. S. Ge, "Design and analysis of a general recurrent neural network model for time-varying matrix inversion," IEEE Transactions on Neural Networks, vol. 16, no. 6, pp. 1477-1490, 2005.

[19] S. Li, S. Chen, and B. Liu, "Accelerating a recurrent neural network to finite-time convergence for solving time-varying Sylvester equation by using a sign-bi-power activation function," Neural Process Lett, vol. 37, pp. 198-205, 2013.

[20] S. Li and Y. Li, "Nonlinearly activated neural network for solving timevarying complex sylvester equation," IEEE Transactions on Cybernetics, vol. 44, no. 8, pp. 1397-1407, 2014.

[21] Y. Shen, P. Miao, Y. Huang, and Y. Shen, "Finite-time stability and its application for solving time-varying Sylvester equation by recurrent neural network," Neural Process Lett, vol. 42, pp. 763-784, 2015.

[22] L. Jin, Y. Zhang, and S. Li, "Integration-enhanced Zhang neural network for real-time-varying matrix inversion in the presence of various kinds of noises," IEEE Transactions on Neural Networks and Learning Systems, vol. 27, no. 12, pp. 2615-2627, 2016.

[23] L. Xiao, Y. Zhang, J. Dai, J. Li, and W. Li, "New noise-tolerant ZNN models with predefined-time convergence for time-variant Sylvester equation solving," IEEE Transactions on Systems, Man, and Cybernetics: Systems, vol. 51, no. 6, pp. 3629-3640, 2021.

[24] M. Zhang and B. Zheng, "Accelerating noise-tolerant zeroing neural network with fixed-time convergence to solve the time-varying Sylvester equation," Automatica, vol. 58, no. 3, 2022, to be published.

[25] L. Shen, J. Wu, and S. Yang, "Initial position estimation in SRM using bootstrap circuit without predefined inductance parameters," IEEE Transactions on Power Electronics, vol. 26, no. 9, pp. 2449-2456, 2011

[26] Z. Zhang, L. Zheng, J. Weng, Y. Mao, W. Lu, and L. Xiao, "A new varying-parameter recurrent neural-network for online solution of timevarying Sylvester equation," IEEE Transactions on Cybernetics, vol. 48, no. 11 , pp. 3135-3148, 2018

[27] Z. Zhang, X. Deng, X. Qu, B. Liao, L.-D. Kong, and L. Li, "A varyinggain recurrent neural network and its application to solving online timevarying matrix equation," IEEE Access, vol. 6, pp. 77 940-77 952, 2018

[28] L. Xiao and Y. He, "A noise-suppression ZNN model with new variable parameter for dynamic Sylvester equation," IEEE Transactions on Industrial Informatics, vol. 17, no. 11, pp. 7513-7522, 2021.

[29] F. Xu, Z. Li, Z. Nie, H. Shao, and D. Guo, "Zeroing neural network for solving time-varying linear equation and inequality systems," IEEE Transactions on Neural Networks and Learning Systems, vol. 30, no. 8, pp. 2346-2357, 2019.

[30] Z. Jiang, Y. Lin, and Y. Wang, "Stabilization of nonlinear time-varying systems: a control Lyapunov function approach," Journal of Systems Science and Complexity, vol. 22, no. 4, pp. 683-696, 2009.

[31] F.-T. Cheng, T.-H. Chen, and Y.-Y. Sun, "Resolving manipulator redundancy under inequality constraints," IEEE Transactions on Robotics and Automation, vol. 10, no. 1, pp. 65-71, 1994.

[32] K. C. Park, P. H. Chang, and S. H. Kim, "The enhanced compact qp method for redundant manipulators using practical inequality constraints," in Proceedings. 1998 IEEE International Conference on Robotics and Automation, vol. 1, 1998, pp. 107-114.

[33] Y. Zhang, M. Yang, D. Chen, W. Li, and X. Yan, "Proposing, QPunification and verification of DLSM based MKE-IIWT scheme for redundant robot manipulators," in Proceedings. 2017 IEEE 3rd Information Technology and Mechatronics Engineering Conference, 2017, pp. 242-248.

[34] F. Xu, Z. Li, Z. Nie, H. Shao, and D. Guo, "New recurrent neural network for online solution of time-dependent underdetermined linear system with bound constraint," IEEE Transactions on Industrial Informatics, vol. 15, no. 4, pp. 2167-2176, 2019. 


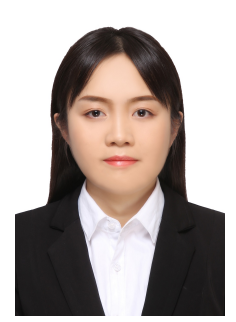

Miaomiao Zhang was born in Laiwu, Shandong, China, in 1997. She received the B.S. degree from the department of Mathematics, Lyuliang University, Lyuliang, China, in 2019. She is currently pursuing her M.S. degree in the School of Mathematics and Statistics, Lanzhou University, China. Her research interests include neural network, perturbation analysis of the matrix and numerical linear algebra.

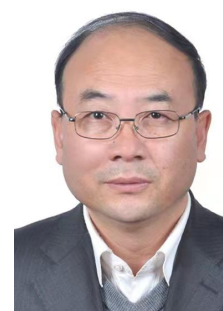

Bing Zheng was born in 1963. He received the M.S. degree in fundamental Mathematics from the School of Mathematical Sciences, Anhui University, China, in 1989, and Ph.D. degree in computational mathematics from the College of Sciences, Shanghai University, China, in 2003. He is currently a full time professor of the School of Mathematics and Statistics at Lanzhou University, China. His research interests include numerical linear algebra and its applications, multilinear algebra, optimization theory, total least squares problem, perturbation analysis of the matrix and so on. He has published more than 90 papers in scientific journals. 